\title{
Exploring divisibility \& summability of 'photon' wave packets in nonlinear optical phenomena
}

\author{
Narasimha Prasad \\ NASA Langley Research Center, 5 N. Dryden St., MS 468, Hampton, VA 23681 \\ narasimha.s.prasad@nasa.gov
}

and

Chandrasekhar Roychoudhuri

Department of Physics, University of Connecticut, Storrs, CT 06269

chandra@phys.uconn.edu

\begin{abstract}
Formulations for second and higher harmonic frequency up \& down conversions, as well as multi photon processes directly assume summability and divisibility of photons. Quantum mechanical (QM) interpretations are completely congruent with these assumptions. However, for linear optical phenomena (interference, diffraction, refraction, material dispersion, spectral dispersion, etc.), we have a profound dichotomy. Most optical engineers innovate and analyze all optical instruments by propagating pure classical electromagnetic (EM) fields using Maxwell's equations and gives only 'lip-service' to the concept "indivisible light quanta". Further, irrespective of linearity or nonlinearity of the phenomena, the final results are always registered through some photo-electric or photo-chemical effects. This is mathematically well modeled by a quadratic action (energy absorption) relation. Since QM does not preclude divisibility or summability of photons in nonlinear \& multi-photon effects, it cannot have any foundational reason against these same possibilities in linear optical phenomena. It implies that we must carefully revisit the fundamental roots behind all light-matter interaction processes and understand the common origin of "graininess" and "discreteness" of light energy.
\end{abstract}

Key words: Photons, light-matter interaction, polarizability, nonlinear optical process, three wave mixing

\section{INTRODUCTION}

Motivation for this paper derives from the following questions: Does indivisible photon picture give any better understanding of the light-matter interaction in nonlinear optical processes? Or is indivisible photon based interpretation a physical reality? In this paper, we will investigate photon picture interpretation in the context of second order nonlinear optical processes.

The era of experimental nonlinear optics began with the invention of laser. It set in motion experimental nonlinear optics. Experimental studies to investigate the behavior of high intensity light beams (optical radiation) in media were carried out with increased peak powers. It enabled the advancement of nonlinear optical processes and allowed new observations such as new optical frequency changes in nonlinear optical media (second order nonlinear process or Three Wave Mixing) and variation of refractive index with optical intensity (self-focusing). It underscored linearity or nonlinearity is a property of the medium and not of the optical radiation. Nonlinear behavior is not exhibited when light travels in free space, even when the intensity is extremely high. Various applications of light-matter interaction or light interacting with light via a medium came to limelight. Nonlinear optical experiments have made profound impact on harnessing energy of photon wave packets for various applications including optical communications, remote sensing, and spectroscopy.

\subsection{Nonlinear Optics}

Nonlinear optics [1-5] deals with interaction between electric field and atomic field. It deals with the effects of non-linear response of a dielectric medium at the atomic level to the electric fields of an intense optical radiation. It encompasses the processes of governing energy transfer between optical fields of 
different frequency or direction as intensity dependent scattering and absorption process. It is observable when electric field amplitudes of optical radiation become comparable with coulomb fields near atoms $\left(\sim 10^{6} \mathrm{~V} / \mathrm{m}\right)$. Nonlinear contributions become important at high optical intensities. Intense radiation produced by a laser propagating through an optical medium produces changes in the spatial and temporal distribution of electric charges which result in the displacement of valence electrons from their normal orbits. This perturbation creates electric dipole moments whose manifestation is the induced polarization, i.e. induced density of electric dipole moment. The electric field of the propagating high intensity optical radiation from a laser causes electric polarization in the medium. This polarization propagates together with the electromagnetic field in the form of a polarization wave.

Nonlinear processes generally do not require the assumption of photons. As such, they are modeled by treating atoms as nonlinear oscillators without any new quantization process. The nonlinear oscillator behavior is described by Lorentz oscillator model for the equation of motion for an atomic electron in an electric field $\mathrm{E}(\mathrm{t})$. A perturbative solution of the nonlinear oscillator equation is sought.

In the case of light-matter interaction, light is generated or absorbed via undulation of material dipoles. We are exploring whether the indivisible photon picture provide a better understanding of nonlinear optical processes. All material media under strong stimulation by EM waves will display both linear and nonlinear properties but the strength of the nonlinear property will depend upon the strength of the stimulation. When stimulation frequency matches with quantum transition, the linear processes dominate. Response of bulk medium without quantized energy levels corresponds to classical nonlinear optics as in the case of second-order nonlinear optics or three wave mixing. When the quantum energy levels of the atoms and molecules are involved, then the process is quantum mechanical in nature as in Raman scattering and twophoton Fluorescence). Our focus in this paper is the classical second order nonlinear optical processes, a bulk phenomenon

\section{LIGHT INDUCED POLARIZATION}

The propagating light in a medium induces polarization as described by Maxwell's equation. The relation between $\mathrm{P}$ and $\mathrm{E}$ is nonlinear. Expanding the $\mathrm{P}$ and $\mathrm{E}$ relationship using Taylor's Series, we can write

$$
\mathbf{P}=\varepsilon_{0} \cdot \chi \cdot \mathbf{E}+\mathbf{2} \cdot \overline{\mathbf{d}} \cdot \mathbf{E}^{2}+4 \cdot \chi^{(3)} \cdot \mathbf{E}^{3}+\ldots
$$

Where $\varepsilon_{0}$ is Permittivity of Free Space, $\chi$ is the Electric Susceptibility of the medium (=Scalar Constant), and $E$ is the Electric Field , $[\mathrm{V} / \mathrm{m}]$. The vectors $P$ and $E$ at any position and time are parallel and proportional. The first term, $\varepsilon_{0} . \chi . \mathrm{E}$, in Eq. 1 represents linear dielectric medium. The second and subsequent terms collectively represents the nonlinear dielectric medum, the effects of which attain significance. The term 2.d. $\mathrm{E}^{2}$ is the second order nonlinearity where $\mathrm{d}$ is the nonlinear optical coefficient that is used instead of $\chi^{(2)}$ for practical reasons. The Polarization density (Dipole moment per unit volume), $P$ is given by $\mathbf{P}=\mathrm{N} \cdot \mathrm{e} \cdot \mathbf{x}$, where $\mathbf{N}=$ Number of molecules per unit volume, $e=$ electric charge and $\mathbf{x}=$ electron displacement.

The second (lowest) order of nonlinear polarization can arise from a $\chi^{(2)}$ nonlinearity which can occur only in crystal materials with a non-centrosymmetric crystal structure. The nonlinear polarization then has a component which depends quadratically on the electric field of an incident light wave. The nonlinear polarization contains frequency components which are not present in the exciting beam(s). Second Harmonic Generation (SHG), Optical Parametric Amplification (OPA), Sum Frequency generation (UpConversion) (SFG), and Optical Parametric Oscillation (OPO). Parametric processes are usually polarization-dependent: the nonlinearity itself is polarization-dependent, and at least in cases with a $\chi^{(2)}$ nonlinearity also the phase matching, because such media exhibit birefringence.

Third-order Nonlinear Polarization allows four-wave mixing processes. The next higher order of nonlinear polarization can arise from a $\chi(3)$ nonlinearity that give rise to various phenomena: The Kerr effect is a nonlinear modification of the refractive index which leads to phenomena such as self-phase modulation, cross-phase modulation, Kerr lensing and four-wave mixing. A delayed nonlinear response is associated with Raman scattering and Brillouin scattering. 
All linear and nonlinear oscillations takes place under any stimulation by light waves, only the strength of the nonlinear oscillations are extremely weak. Notice that there is no threshold limit that bars higher order stimulations. The $\chi^{(\mathrm{n})}$ terms being weak, we need high intensity fields, normally generated by lasers.

Under any stimulation all linear and nonlinear oscillations takes place. Notice that there is no threshold limit that bars higher order stimulations - just a matter of strength of stimulating fields. But the $\chi^{(n)}$ terms being weak, we need high intensity fields normally generated by lasers.

The electric field of light can be represented by

$$
\mathbf{E}(\mathbf{t})=\mathbf{E}_{\mathbf{0}} \cdot \cos (\boldsymbol{\omega} \cdot \mathbf{t})
$$

From Eq. 1, for second order effect,

$$
\text { Polarization } \propto E^{2}(t)=\mathbf{E}_{0}^{2} \cdot \cos ^{2}(\omega \cdot t)=\frac{1}{2}\left[E_{0}^{2}+E_{0}^{2} \cdot \cos ^{2}(2 \omega \cdot t)\right.
$$

For three wave mixing, we have two fields, $\mathrm{E}_{1}(\mathrm{t})$ and $\mathrm{E}_{2}(\mathrm{t})$. Hence,

$$
E(t)=\left[E_{1} \cdot \cos \left(\omega_{1} \cdot t\right)+E_{2} \cdot \cos \left(\omega_{2} \cdot t\right)\right]
$$

Then,

$$
\begin{aligned}
E^{2}(t)= & {\left[E_{1} \cdot \cos \left(\omega_{1} \cdot t\right)+E_{2} \cdot \cos \left(\omega_{2} \cdot t\right)\right]^{2} } \\
=\frac{1}{2}[ & E_{1}^{2}+E_{2}^{2}+E_{1}^{2} \cdot \cos ^{2}\left(2 \cdot \omega_{1} \cdot t\right)+E_{2}^{2} \cdot \cos ^{2}\left(2 \cdot \omega_{2} \cdot t\right) \\
& \left.+E_{1} E_{2} \cdot \cos \left(\omega_{1}+\omega_{2}\right) \cdot t+E_{1} E_{2} \cdot \cos \left(\omega_{1}-\omega_{2}\right) \cdot t\right]
\end{aligned}
$$

The nonlinear component of the polarization, $\mathrm{P}_{\mathrm{NL}}=2 \cdot \mathrm{d} \cdot \mathrm{E}^{2}$ contains components $0,2 \omega_{1}, 2 \omega_{2},\left(\omega_{1}+\omega_{2}\right),\left(\omega_{1}-\omega_{2}\right)$. Alternately, we can write,

$$
\mathbf{E}(\mathbf{t})=\mathbf{E}_{\boldsymbol{\theta}} \cdot \cos (\omega \cdot t)=\operatorname{Re}\left\{\delta_{\omega} \cdot \mathrm{e}^{-1 \omega t}\right\}=\frac{1}{2} \cdot\left(\mathcal{E}_{\omega} \cdot \mathrm{e}^{-1 \omega t}+\tilde{\sigma}_{\omega}^{\cdot} \cdot \mathrm{e}^{\mathrm{l} \omega t}\right)
$$

Writing,

$$
\mathcal{E}_{\omega}=\mathcal{E}_{\omega}(z) \cdot \mathbf{e}^{i k_{\omega} z}
$$

$$
\mathbf{E}(\mathbf{t})=\operatorname{Re}\left\{\mathcal{E}_{\omega} \cdot \mathrm{e}^{-\mathrm{i}\left(\mathbf{k}_{w} z-\omega t\right)}\right\}=\frac{1}{2} \cdot\left\{\mathcal{E}_{\omega} \cdot \mathrm{e}^{-\mathrm{i}\left(\mathbf{k}_{w} z-\omega t\right)}+\mathcal{E}_{\omega}^{\cdot} \cdot \mathrm{e}^{\mathrm{i}\left(\mathbf{k}_{w} z-\omega t\right)}\right\}
$$

For three wave mixing process, we have two harmonic components namely,

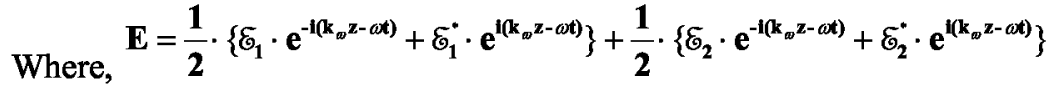

$$
\begin{aligned}
& \mathbf{k}_{\mathbf{1}}=\frac{\mathbf{n}\left(\omega_{i}\right) \omega_{i}}{\mathbf{c}}
\end{aligned}
$$

Writing,

$$
\mathbf{E}_{3}=\frac{1}{2} \cdot\left\{\mathcal{E}_{3} \cdot \mathbf{e}^{-\mathbf{i}\left(\mathbf{k}_{\omega} z-\omega t\right)}+\mathcal{E}_{3}^{*} \cdot \mathbf{e}^{\mathrm{i}\left(\mathbf{k}_{\omega} \mathbf{z}-\omega t\right)}\right\}
$$

We obtain three coupled equations that can be succinctly written as,

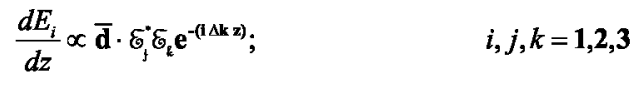

For second order nonlinear processes, the induced polarization is proportional to 


$$
\chi^{(2)} E^{2}(t)=\chi^{(2)} \cdot \mathbf{E}_{0}^{2} \cdot \cos ^{2}(\omega \cdot \mathbf{t})
$$

The polarization is material dipole undulation. Expanding the cosine function,

$$
\chi^{(2)} E^{2}(t)=\frac{1}{2} \cdot \chi^{(2)} \cdot\left[\mathbf{E}_{0}^{2}+\mathbf{E}_{0}^{2} \cdot \cos (2 \omega \cdot \mathbf{t})\right]
$$

In the above equation, the first term represents the DC polarization and the second term represents the sinusoidal oscillation. This sinusoidal undulation, when in phase with the fundamental wave, can convert a part of the fundamental energy into the new second harmonic radiation. This is a bulk dipole undulation process and the energy conversion efficiency depends upon the physical length over which the phase matching condition remains valid. The walk-off and other associated issues arise when the wave-front vector and the Poynting vector cannot remain collinear for a given crystalline medium. Maxwell's equation clearly implies that the light emitting sources have to execute sinusoidal oscillations to be able to emit sinusoidal light waves. Materials cannot generate cosine squared radiation. Eq.1-13 has no quantum mechanical phenomenon built into them. The DC polarization term is purely a classical bulk phenomenon.

\section{PHASE MATCHING AND WALK-OFF}

Phase matching provides maximum and sustained harmonic conversion over physical length of the nonlinear medium. The nonlinear mixing products can be efficiently accumulated over a greater length of crystal only if phase matching is achieved. Because of differential variations in the refractive indices, it is possible that the phase can be reversed and the non-linear radiation can get converted back into the fundamental stimulating radiation. Some nonlinear effects, however, are either automatically phasematched (e.g. self-phase modulation) or do not require phase matching (e.g. Raman scattering). Many phase-sensitive nonlinear processes, in particular parametric processes require phase matching to be very precise. Essentially, this means ensuring that a proper phase relationship between the interacting waves (for optimum nonlinear frequency conversion) is maintained along the propagation direction, so that the amplitude contributions from different locations to the product wave are all in phase and hence phase mismatch has to be close to zero.

For three-wave mixing processes, phase matching conditions involve frequency and momentum matching relations. The frequency matching condition is given by

$$
\omega_{3}=\omega_{1}+\omega_{2}
$$

The phase or momentum matching condition in terms of wave vectors is given by

$$
\overrightarrow{\mathbf{k}}_{\mathbf{3}}=\overrightarrow{\mathbf{k}_{1}}+\overrightarrow{\mathbf{k}_{2}}
$$

In the case of optical parametric oscillations, the following cavity resonance conditions in terms of cavity length $\mathrm{L}$, speed of light, $\mathrm{c}$, and integers $\mathrm{N}_{1}$ and $\mathrm{N}_{2}$ have to be satisfied for signal and idler wavelengths.

$$
\omega_{1}=\left(N_{1} \frac{\pi \cdot c}{n_{1} L}\right) \& \omega_{2}=\left(N_{2} \frac{\pi \cdot c}{n_{2} L}\right)
$$

Integration of Eq. 11 using slowly varying approximation provides field amplitudes of electric field amplitudes. The electric field amplitudes are related to phase mismatch, $\Delta \mathrm{k}$ via a Sinc function shown below.

$$
\begin{aligned}
& \mathbf{E}_{\mathbf{i}} \propto \frac{\sin ^{2}\left(\frac{\Delta \mathbf{k}}{2} z\right)}{\left(\frac{\Delta \mathbf{k}}{2} z\right)^{2}} \\
& \text { where, } \Delta \mathbf{k}=\mathbf{k}_{1}+k_{2}-k_{3} ; \text { Phase Mismatch }
\end{aligned}
$$

The term coherence length $\mathrm{L}_{\mathrm{c}}=\pi /(\Delta \mathrm{k})$, is often used to describe the interaction length when it decreases to $40 \%$ of its peak value. Finally, Manley-Rowe Relation in Eq. 18 for three wavelengths describes energy conservation. 


$$
\frac{d}{d z}\left[\frac{I_{3}}{\omega_{3}} \mid\right]=\frac{d}{d z}\left[\left.\frac{I_{1}}{\omega_{1}}\right|_{\rfloor}=\frac{d}{d z}\left[\left.\frac{I_{2}}{\omega_{2}}\right|_{]}\right.\right.
$$

\section{Phase-matching Techniques-A Brief Review}

The common technique for achieving phase matching in nonlinear crystals is birefringent phase matching based on crystal birefringence. The birefringent technique to appropriately compensate for phase velocity mismatch comes in many variations. In the case of second order nonlinear effect (or three wave mixing), three beams are involved namely, pump, signal and idler. Optical parametric oscillation can be represented by pump $=$ signal + idler indicating signal (lower wavelength) and idler (higher wavelength) beams are generated from the pump beam. In this context, Type I phase matching is given by $\mathbf{e}=\mathbf{o}+\mathbf{o}$ relation where pump beam corresponds to extra-ordinary wave (e) and signal and idler beams correspond to ordinary wave (o). Similarly, Type II Phase matching is given by $\mathrm{e}=\mathrm{o}+\mathrm{e}$ for the three beams. Besides desired signal wavelength and availability of pump beam wavelength, bulk (physical and birefringence) properties of the nonlinear medium dictate the type of phase matching to be chosen for efficient phase matching process. Critical phase matching is referred to angular alignment of the crystal (or the beam) is used to find a phase-matching configuration. Noncritical phase matching refers to all polarization directions are along the crystal axes. The angular position is then not a sensitive parameter and the phase mismatch is minimized by adjusting the crystal temperature such that the phase velocities of the interacting beams are equal. This technique avoids spatial walk-off that will be discussed later. Also, if the wave vectors of all involved beams have the same direction it is referred to as collinear phase matching. In the case of different directions, it is known as noncollinear phase matching, In any case, the vector sum of the generating beams equals the wave vector of the product beam. A special case is achromatic phase matching where at least one of the interacting beams is angularly dispersed so that each frequency component of the signal is properly phase-matched. In certain cases, phase matching doesn't occur or even if it occurs it may not be efficient in a given crystal direction due to low nonlinear figure of merit. In such cases, Quasi-phase matching technique is employed using engineered materials. In this case, the sign (or strength) of the nonlinearity varies periodically and obtained by poling techniques.

In the context of phase matching, group velocity mismatch and bandwidth limitations assumes significance. Under phase matching condition the group velocities of the interacting waves are, in general, still not matched; there exists a certain group velocity mismatch, which limits the interaction length for pulses and (for a given interaction length) the spectral range (called phase-matching bandwidth) in which phase matching is achieved. Also, there is only a finite range of beam angles where phase matching works - particularly for critical phase matching called as the angular phase-matching bandwidth

\section{Walk-off}

Walk-off phenomenon assumes significance in second order nonlinear processes limits harmonic conversion efficiency [6]. Spatial Walk-off - the phenomenon that the intensity distribution of a beam in an anisotropic crystal drifts away from the direction of the wave vector.

In an isotropic medium, the transverse intensity distribution propagates along the beam axis as defined by the medium $k$ vector whereas in anisotropic (and thus birefringent) crystals, the intensity distribution drifts away from the $k$ vector direction. The Poynting vector defines the direction of energy transport, whereas the $k$ vector is normal to the wavefronts. This phenomenon, called spatial walk-off, birefringent walk-off or Poynting vector walk-off (not to be confused with temporal walk-off), is associated with some finite angle $\rho$ (called walk-off angle) between the Poynting vector and the $k$ vector

$$
\rho=-\frac{1}{n_{e}} \frac{d n_{e}}{d \theta}
$$

Phase matching and Walk-off are purely classical phenomenon based on bulk material properties of crystals and doesn't require the use of any quantum mechanical formulations or concepts (use of Schrodinger Equation, $2^{\text {nd }}$ quantization of EM wave fields, energy transitions levels, etc.). 


\section{Photon Picture: As described in the standard literature}

In quantum physics, photon is a quantized elementary particle, the quantum of the electromagnetic field and the basic "unit" of light (or all forms of electromagnetic radiation). Some times it is described as a monochromatic mode of the vacuum with energy $h v$, where $v$ is the uniquely defined frequency. Photons are considered stable Bosons with zero rest mass, zero electric charge and unit spin. Being Bosons, photons occupy same space without interfering with each other [7 ].

\section{Photons and Nonlinear Optics Processes-A discussion}

Often in literature, people invoke quantum mechanics to describe nonlinear optical processes. Figure 1 illustrates SHG and down conversion cases. However, illustrations in Figure 1 are extended to other three wave mixing processes. Nonlinear optical processes do not require the assumption of photons hence are often modeled by treating atoms as nonlinear oscillators.

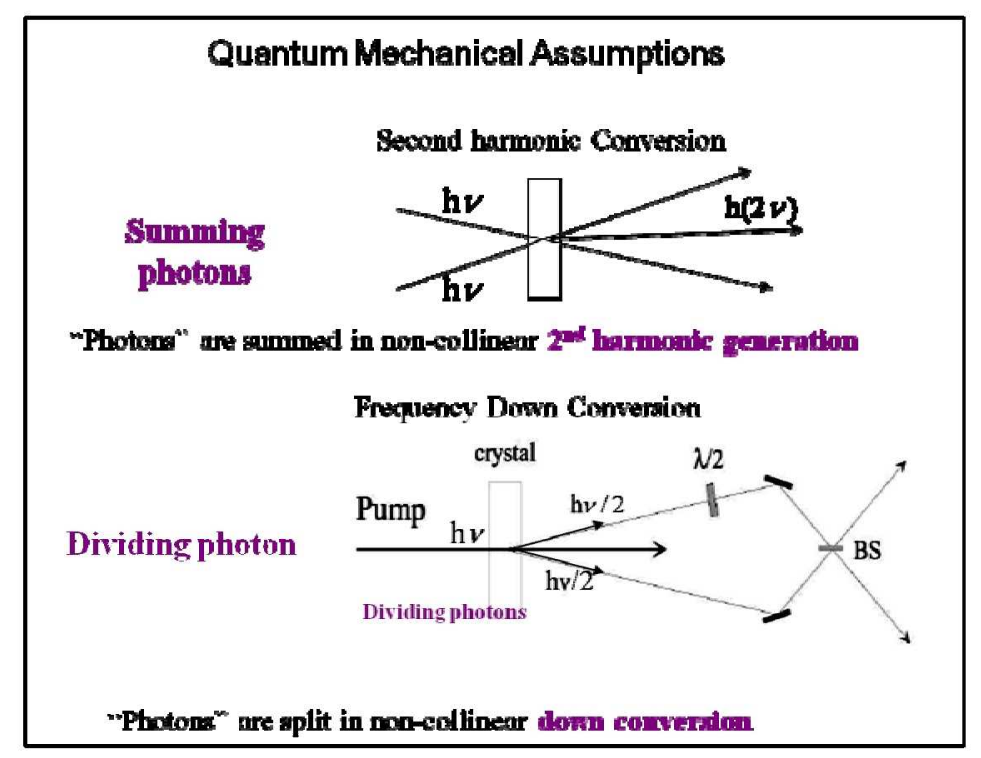

Figure 1. Quantum mechanical assumptions used in discussing nonlinear optical processes.

\section{Photon Based Interpretation - Mathematical formalism or physically reality?}

Often people invoke photon concepts to explain nonlinear wave mixing processes. Is the term Three Photon Interaction or mixing a physical reality?

Classical frequency and phase matching equations given in Eq.14 \& 15 are often re-written by multiplying them through out by $(\mathrm{h} / 2 \pi)$ as:

$$
\begin{aligned}
& {\left[\frac{h}{2 \pi}\right]^{\prime} \omega_{3}=\left[\frac{h}{2 \pi} \mid\right]^{\omega_{1}}+\left[\frac{h}{2 \pi}\right]^{\prime} \omega_{2}} \\
& \left.\left.\left[\frac{h}{2 \pi} \mid\right]^{\mid} \vec{k}_{3}=\left[\frac{h}{2 \pi}\right]\right]^{\mid} \vec{k}_{1}+\left[\frac{h}{2 \pi}\right]\right]^{\vec{k}_{2}}
\end{aligned}
$$

Also, Manley Rowe relation [Eq.18] describing conservation of energy is written in terms of photon number conservation based on photon flux densities (photons $/ \mathrm{s}-\mathrm{m}^{2}$ ) 


$$
\frac{d \phi_{1}}{d z}=\frac{d \phi_{2}}{d z}=-\frac{d \phi_{3}}{d z}
$$

The equality of classical relations are maintained while by multiplying appropriate constants both sides and hence to create a quantum mechanical interpretation. This is an ad hoc approach to convert purely classically derived relations without any physical explanations as to why one needs to make them look like quantum mechanical. The experimental validity of Eq.20-22, or any equation, of course, will remain intact when they are multiplied throughout by the same constant. But this process does not help us understand the nonlinear processes any deeper than what the classical mathematical relations, Eq.14, 15 \& 18 have provided us. Nonlinear optical processes are classical bulk phenomena.

Photons being non-interacting Bosons, photons can occupy same space at the same time and photonphoton interaction is negligible. How can a stable elementary particle undergo self-interference? Optical radiation of terawatt levels can be focused through a pin hole. In nanophotonics, $600 \mathrm{~nm}$ wavelength light beams have been propagated through glass fiber of $\sim 100 \mathrm{~nm}$ diameter which implies photons can be "compressed" from a $1 \mathrm{~mm}$ beam into a $1 \mathrm{~nm}$ beam. All these properties are quantitatively and very precisely modeled by classical wave equation and Huygens-Freslnel's diffraction theory (and consequent laser beam propagation theory). Introduction of indivisible photon model not only does not bring out any better understanding of the physical processes behind nonlinear optics, it creates further confusion in distinguishing between real quantum phenomena like Raman Effect and multi-photon atomic processes and pure classical bulk crystalline processes. The key point is that without light-matter interaction, and mediated by collective dipole undulations of bulk material and stimulated by incident "fundamental frequency" of light, we cannot get new optical frequencies. Only material dipoles can create or annihilate E.M radiation. Again, photons being Bosons, cannot interact with each other to create new photons. Researchers are looking into various predispositions of light-matter interactions to understand true nature of photons [8-11].

\section{Quantum and Bulk Phenomenon}

Quantum theory allows discrete energy level transitions. In traditional nonlinear optical processes (frequency up or down conversion using crystals), no quantum mechanically defined energy-band transitions or discrete energy level transitions are involved. Continuous energy transfer from primary frequency to secondary frequency or pump to signal and idler takes place over long distances in bulk medium inside the nonlinear crystal when phase matching conditions are satisfied. $\chi$ represents bulk characteristics. Quasi-phase matching allows energy conversion over increased lengths. Maker fringes confirm the phase matching process. As discussed earlier, walk-off is due to dispersion, pump and signal or idler energy vector separation. Walk off is a bulk crystal property. During the energy transfer process, if the phases are reversed $\left(180^{\circ}\right.$ apart), back conversion takes place (signal and idler energies converts back into pump) which is easily understood by classical physics. Walk off and Back Conversion are not Quantum mechanical processes. Accordingly, frequency summation and momentum vector summation is not a justification to invoke bulk nonlinear processes as a quantum mechanical phenomena. These equations are representative formalisms with no underlying Quantum Mechanical physics. In contrast, Fig.2 provides well known examples of linear and nonlinear quantum mechanical processes.
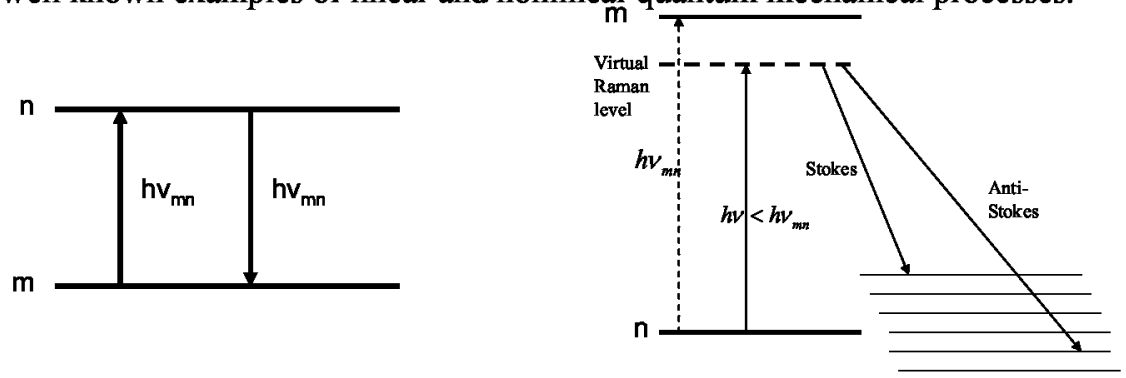

Figure 2. Linear and Nonlinear Quantum mechanical processes. Left - linear resonance fluorescence. Right - nonlinear (Raman) molecular transitions. 


\section{CONCLUSIONS}

Only light-matter interaction process can generate new frequencies. Bulk crystalline dipole undulation via light-matter interaction allows new frequencies to be generated. The processes could be quantum mechanical or classical and could be linear or non-linear. We clam that the traditional nonlinear optical processes to generate up- or down-converted new optical frequencies are best explained by classical formulation that uses bulk material properties. Conversion efficiency depends upon the strength of the stimulating field in the presence of effective phase matching or over long coherence lengths. Phase matching and walk-off are classical phenomena. Ad hoc multiplications through out of certain classically derived equations with suitable constants to make them look like quantum mechanical relations, do not bring out any better understanding of the underlying physical light-matter interaction processes.

\section{REFRENCES}

1. D. A. Kleinman, "Nonlinear dielectric polarization in optical media", Phys. Rev. $126(6), 1977(1962)$

2. G. D. Boyd and D. A. Kleinman, "Parametric interaction of focused Gaussian light beams", J. Appl. Phys. 39 (8), 3597 (1968)

3 P.W. Milonni and J.H. Eberly, Lasers, John Wiley and Sons, Inc., New York, 1988

4 A.Yariv and P.Yeh, Optical Waves in Crystals, John Wiley and Sons, Inc., New York, 1983

5 B.E.A Saleh and M.C. Teich, Fundamentals of Photonics, John Wiley and Sons, Inc., New York, 1991

6 A. V. Smith et al., "Increased acceptance bandwidths in optical frequency conversion by use of multiple walk-off-compensating nonlinear crystals", J. Opt. Soc. Am. B 15 (1), 122 (1998); see also references therein

7 Photon in Wikipedia (see other references cited in this article)

8 C. Roychoudhuri and N. Prasad "Various ambiguities in re-constructing laser pulse parameters", proceedings of the October, 2006 IEEE-LEOS Annual Conference, Montreal, Canada; Invited Talk.

9 C. Roychoudhuri, see Chs.6, 24 \& 25 in The Nature of Light: What Is a Photon? Eds. C. Roychoudhuri, A. F. Kracklauer \& Kathy Creath; CRC Press (2008).

10 C. Roychoudhuri and P. Poulos, SPIE. Proc. Vol.6664 (2007); The Nature of Light: What Are Photons?; doi: 10.1117/12.740177; "Can we get any better information about the nature of light by comparing radio and light wave detection processes?"

11 C. Roychoudhuri, A. F. Kracklauer \& Kathy Creath, The Nature of Light: What Is a Photon?; CRC (2008); see Chapters 24, 25, $26 \& 6$. 